\title{
COMET HALLEY: A CARRIER OF INTERSTELLAR DUST CHEMICAL EVOLUTION
}

\author{
J. Mayo Greenberg \\ Laboratory of Astrophysics, University of Leiden, Niels Bohrweg 2, \\ 2333 CA Leiden, The Netherlands
}

\section{ABSTRACT}

Cometary material, as deduced from comet Halley results, bears a remarkable resemblance to the chemical and morphological structure of a primitive aggregate of interstellar dust. The principal clues are found in 1) the sizes of cometary dust, 2) the non volatile organics in cometary dust, 3) the surface albedo, 4) the physical and photochemical break-up of the dust molecules in the coma via the appearance, for example, of $\mathrm{CN}$ and very abundant $\mathrm{C}^{+}$ions. 5) the high surface temperature but low internal temperature. 6) low comet density. 7) solar orientation of gas and dust jets. 8) low $\mathrm{C} / 0$ ratio in coma.

There are several reasons to expect no significant modification of the interior of comet Halley since it was formed $4 \frac{1}{2}$ thousand million years ago. The proven presence of a major complex organic dust component along with the low comet density implied by the low albedo and large size $\rightarrow$ combine to provide further confirmation of the possible contribution of comets to the origin of life.

\section{INTRODUCTION}

The chemical composition and size distribution of interstellar dust has been studied by theoretical interpretation combining remote observations and laboratory experiments /1/. Following the various stages of evolution leads to a picture of grains as consisting of basically two distinct size populations: tenth micron and hundredth (or less) micron. The larger particles are core-mantle structures with cores of silicates, inner mantles of a complex organic refractory material and outer mantles of various ices dominated by frozen $\mathrm{H}_{2} \mathrm{O}$. The very small particles are again of two types one type is mostly carbon, the other is presumed to be made of silicate material. The key to the evolution of grain mantles is solid state photoprocessing while fully taking into account the gas phase and surface reactions of interstellar atoms and molecules /2,3/. After a mean lifetime of several thousands of million years most grains are consumed by star formation. Some, however, remain as the basic building material of solar system bodies and are brought to us at the present time in various stages of preservation since our solar system formed about $4 \frac{1}{2}$ thousand million years ago.

\section{SIZE SHAPE AND COMPOSITION OF INTERSTELLAR DUST}

Recent studies of the observations of soncalled diffuse cloud dust (dust not in molecular clouds) in the ultraviolet have revealed the fact that there are three populations of dust $14,5 /$. There are "large" grains of $-0.15 \mu \mathrm{m}$ in mean radius which provide the major blocking of star light in the visual. The polarization of star light in the visual which is associated with extinction implies that these particles are elongated by at least a factor three /6/. There are also very small carbonaceous particles of $\leq 0.01 \mu \mathrm{m}$ in radius which produce a strong absorption feature at about $220 \mathrm{~nm} / 7 /$. In addition there is an independent population of - $0.01 \mu \mathrm{m}$ silicate type particles.

How the large grains form and evolve is a complex physical and chemical story. Basically they start with silicate particles forming in the atmospheres of cool stars and, after being ejected into space, providing nucleation cores for the growth of mantles of lces which are produced by gas phase and surface reactions of the abundant atomic species, oxygen, carbon, nitrogen and sulfur /8/. These ices are always being photoprocessed by ultraviolet radiation 
from either distant stars or cosmic rays or arising from local hot stars and/or stellar winds. The result of such photoprocessing is not only a change in the basic composition of the ices but also the production of complex organic refractory residues which have been studied in the Leiden laboratory and compared with astronomical observations $/ 9,10 /$.

The direct evidence for organic residues in space was finally exhibited in the observation of a $3.4 \mu \mathrm{m}$ feature seen towards objects in the galactic center $/ 11 \%$. Laboratory produced residues have been successful in recreating the essential shape of the $3.4 \mu \mathrm{m}$ feature which consists of contributions from the $\mathrm{C}-\mathrm{H}$ stretch in $\mathrm{CH}_{2}$ and $\mathrm{CH}_{3}$ groups in complex organic molecules $/ 12,13 /$.

The spectral correspondence between the laboratory residue and the galactic center is supported quantitatively by the measured strength of the laboratory 3.4 um feature $/ 14 /$.

The evolutionary plcture of dust which is emerging is a cyclic one in which the particles find themselves alternately in diffuse clouds and in molecular clouds. A small silicate core captured whithin a molecular cloud gradually builds up an inner mantle of organic refractory material which has been produced by photoprocessing of the volatile ices. Within the dense clouds critical densities lead to star formation and subsequent ejection of some of the cloud material back into the surrounding space. Much of this material finding itself in a very tenuous low density environment expands to the diffuse cloud phase. Dust particles in the diffuse medium are subjected to numerous destructive processes which rapidly erode refractory material. Silicate coretorganic refractory mantle grains survive the diffuse cloud phase to reenter the molecular cloud phase.

The mean star production rate of $1-2 \mathrm{M}_{0} \mathrm{yr}^{-1}$ implies an interstellar mediun turnover time of $-5 \times 10^{9} \mathrm{yr}$ so that this is the absolute maximum lifetime of a dust particle no matter how resistant to destruction. If we use a mean molecular cloud-diffuse cloud period of $2 \times 10^{8} \mathrm{yr}\left(10^{8}\right.$ years in each) then a typical grain anywhere in space will have undergone at least 20 cycles so that, for example, the typical diffuse cloud dust particle age is $210^{9} \mathrm{yr}$ and consists of a mix of particles which have undergone a wide variety of photoprocessing.

The ultraviolet energy absorbed by a typical $0.15 \mu \mathrm{m}$ core-mantle grain in standard energy dose units of rads $\left(1 \mathrm{rad}=100 \mathrm{erg} \mathrm{\textrm {g } ^ { - 1 }}\right)$ is $0.8 \mathrm{rad} \mathrm{s}^{-1}$ while the dose rate on a very small a$0.01 \mu \mathrm{m}$ particle is about ten times higher. Note that the organic refractory mantles are subjected to the highest photoprocessing rates in the diffuse cloud phase. This would imply that the organic refractory mantle on a grain is not a homogeneous substance. A result of sequential formation (in the molecular cloud phase) and of intense photoprocessing (in the diffuse cloud phase) would lead to a layering in which the innermost layers have been the most irradiated and the outermost layer is first generation organic refractory. Because of this kind of layering, and the fact that the grains are of varlous ages leads one to expect average homogenelty of diffuse cloud grains both in size and structure which is observed as a uniformity in the visual extinction curve and a rather structureless $3.4 \mu \mathrm{m}$ feature. In other words, diffuse cloud grains represent a steady state average of grains of a multiplicity of chemical and physical histories.

A schematic representation of grains in the various regions of space is shown in Fig. 1. In the final stage of cloud condensation we may expect that all remaining (condensable) molecules will have accreted onto the dust. In addition, the very small ( $\leq 0.01 \mu \mathrm{m})$ particles will be collected and trapped within the outer volatile icy mantle. In fact, in the dense protosolar cloud, the hundredth micron particles may themselves accrete icy mantles before being collected on the larger grains.

The lack of well-crystalyzed carbon in meteorites $/ 15 /$ would seem to preclude graphite as a substantial component of the interstellar medium. Certainly not as much as the $\sim 32 \%$ of all carbon required in the form of graphite to give the $220 \mathrm{~nm}$ hump $/ 16,17 \%$. A purely interstellar process leading to the presence of a carbon component follows from ultraviolet photoprocessing of grain mantle ices $118,19 \%$. A factor in favor of some interstellar produced organic particles contributing to the $220 \mathrm{~nm}$ hump is the degree of correlation between the visual extinction and the $220 \mathrm{~nm}$ hump $/ 16,20 \%$. In other words we might expect a visual-hump correlation if the "visual particles" can be the source of the "220 nm particles". 

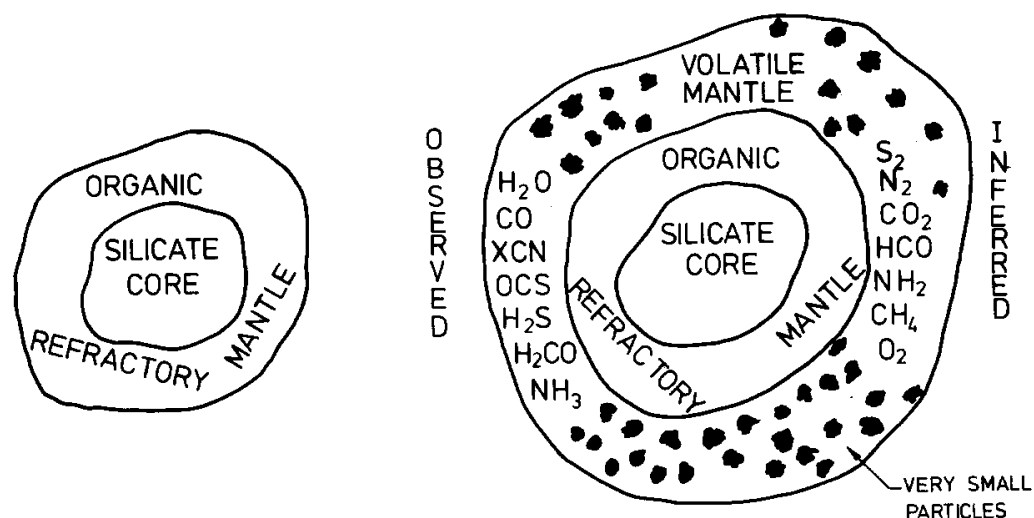

DIFFUSECLOUD GRAIN

PRECOMETARY GRAIN

Fig. 1: Cross sectional structure of interstellar grains in diffuse interstellar clouds and in the protostellar nebula.

However, we must at the outset point out that the organic refractory mantles as such can not be responsible for the ultraviolet absorption feature. This is because the mean particle sizes producing the visual extinction relative to $220 \mathrm{~nm}$ are such that $2 \pi a / \lambda>1$ and the extinction in the ultraviolet is at the saturation level. As a consequence, any extra absorption in the mantle material be unnoticeable if it is superposed on an already significant continuum absorptivity.

It has been proposed that certain linear unsaturated carbon chain molecules have extremely strong absorption in the $200 \mathrm{~nm}$ region. In Leiden we have, in matrices, produced these absorptions by ultraviolet photolysis of a number of starting organic molecules - either hydrocarbons or those containing oxygen or nitrogen. The stable end products appear from infrared and ultraviolet spectrum to be linear chain molecules of the polyacetylene and cyanopolyacetylene type. The assumption here is that some of the organic refractory mantles are either broken off by some grain destruction process, such as shocks, or ejected by grain explosions, and appear in space as very small carbonaceous particles. Subsequently, because of their very small size, the effectiveness of penetrating ultraviolet photons in photolyzing the hydrogen away is enhanced relative to that of the mantle of the core mantle grains where the cage effect is more important. Furthermore, as already noted the absorbed energy dose rate of the small particles is higher than that of the core-mantle particles by a factor of ten. Thus a sufficiently large mass fraction may be converted into chain molecules in the small particles so that they may become efficient "hump" particles. Although a strong "isolated" absorption in the mantles would lead to a distortion in the hump shape, this may be precluded by the existence of a moderate continuum absorptivity underlying the hump feature.

As a cyclic phenomena the $220 \mathrm{~nm}$ particles are accreted along with the icy mantles in the molecular cloud phase $/ 8 /$ and as a result of oxydation and other photochemically induced chemlcal reactions in the solid which is oxygen rich they are incorporated into the mantle material as non $220 \mathrm{~nm}$ absorbing matter. The observed $220 \mathrm{~nm}$ decrease in strength in molecular clouds $/ 21 /$ is consistent with this as well as the fact that even if small organic refractory particles are ejected from manties as a result of explosions, there is not enough ultraviolet 
to convert them to "hump particles" before they are reincorporated in the mantles $/ 22 /$. Regeneration of the hump particles then occurs when the particles reappear in the diffuse cloud phase.

Grain modelling of the far ultraviolet part of the extinction curve has been successfully performed using very small silicate particles. There is no direct evidence for such a population except, as we shall see, perhaps in the mass spectra of the comet Halley dust $123,24 /$.

\section{DUST AGGREGATION AND MORPHOLOGY}

In our solar system all of the planets and satellites have incorporated into their bodies at least the refractory components of the interstellar dust which existed in the pre-solar nebula. In general, the direct connections to the dust have been lost. Comets, are the most likely bodies to have preserved their original composition but the first important question is did their original composition include the volatile as well as the non-volatile components of dust? As is shown elsewhere /25/ the existence of $S_{2}$ as a parent molecule in comets may be traced back to the photochemical evolution of the interstellar dust ice (predominantly $\mathrm{H}_{2} \mathrm{O}$ ) mantles which are known to countain sulfur bearing molecules. Secondly an upper limit on the temperature of formation of the comet is provided by the fact that if the predominantly $\mathrm{H}_{2} \mathrm{O}$ grain mantles evaporate they release the $S_{2}$ which can not reform in the protosolar nebula and is therefore lost to the comet.

As a first approximation, therefore, we consider a comet nucleus as if its chemical composition and morphological structure are directly related to interstellar dust. Table I shows the relative fractions of the various chemical constituents which have been obtained by an extrapolation from the molecular cloud dust phase $126 \%$. In all cases the full cosmic abundances of the elements are assumed.

Table 1: Suggested mass and volume distribution of the principal chemical constituents of a cometsimal. Amounts in parentheses are for very small particles $(\leq 0.01 \mu \mathrm{m})$

Component Mass fraction volume fraction

$\begin{array}{llc}\text { Silicates } & 0.14(0.07) & 0.06(0.03) \\ \text { Carbon (Garbonaceous) } & (0.06) & (0.03) \\ \text { Nonvolatile complex } & & \\ \text { Organic refractory } & 0.19 & 0.21 \\ \mathrm{H}_{2} \mathrm{O} & 0.20 & 0.28 \\ \mathrm{CO} & 0.03 & 0.04 \\ \mathrm{CO}_{2} & 0.04 & 0.05 \\ \text { Other molecules + radicals } & & \\ \left(\mathrm{H}_{2} \mathrm{CO}, \mathrm{HCN}, \mathrm{CN}, \mathrm{N}_{2}, \mathrm{HCO} \ldots\right) & 0.27 & 0.31\end{array}$

In forming the nucleus we assume that first clumps of grains form and then clumps of clumps and so on until finally we reach the size of the comet nucleus. The question is, does this lead to a fully compact structure or does it lead to an open structure. The evidence we have for an open structure is the low density of meteors which are known to be comet debris $127,28,29 \%$. If we should start with the interstellar dust tightly packed and then remove all the volatiles (along with the trapped super small particles) the resulting mean density of the remaining core-organic refractory grains skeleton is about $0.5 \mathrm{~g} \mathrm{~cm}^{-3} / 30 /$. It is however observed that meteors (which are what is left after the original cometary volatiles have evaporated) have a characteristic density of $0.2 \mathrm{~g} \mathrm{~cm}^{-3}$ we see therefore that the original 
dust was not fully packed and, in fact, at most about $40 \%(0.2 / 0.5)$ of the original comet nucleus was filled with dust - the remaining $60 \%$ being empty space. An even lower dust packing factor of about 0.2 is suggested by the mean comet density of $\rho=0.25 \mathrm{~g} \mathrm{~cm}^{-3}$ derived from the tidal splitting of $\mathrm{P} /$ Comet Brooks 2 by Sekanina and Yeomans $/ 31 \%$ A model of such an open aggregate of 100 typical precometary grains is shown in Fig. 2.

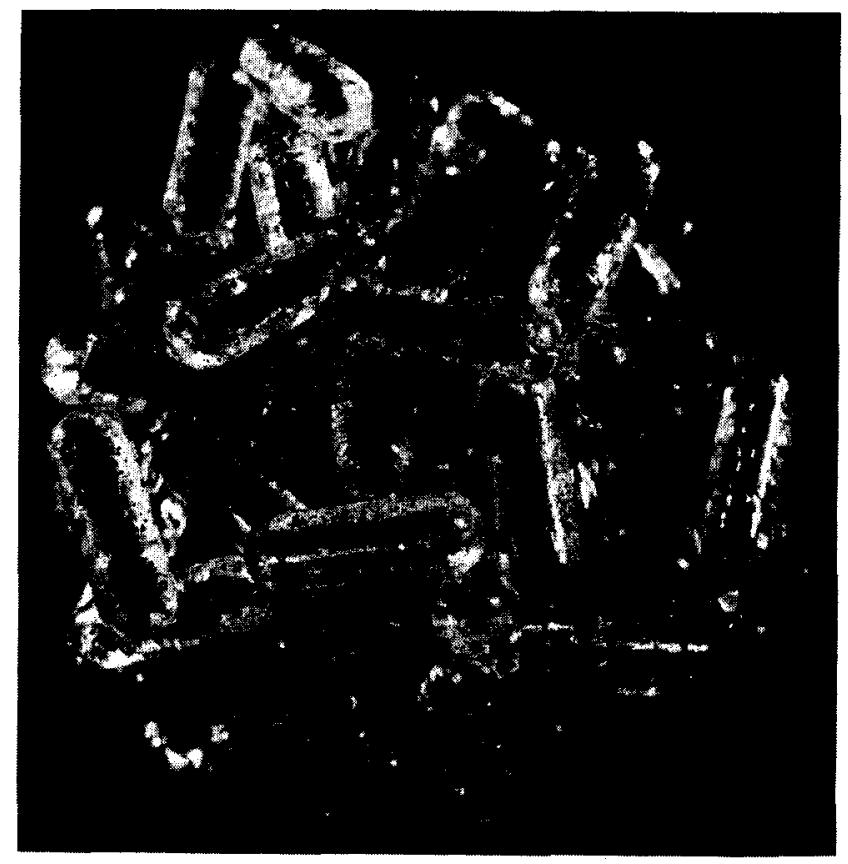

Fig. 2. A PIECE OF A FLUFFY COMET: Model of an aggregate of 100 average interstellar dust particles each of which consists of a silicate core, an organic refractory inner mantle and an outer mantle of predominantly water ice in which are embedded the numerous very small $(<0.01 \mu \mathrm{m})$ particles responsible for the interstellar $216 \mathrm{~nm}$ absorption and the far ultraviolet extinction. Each particle as represented corresponds to an interstellar grain $1 / 2 \mu \mathrm{m}$ thick and about $1 \frac{1}{2} \mu \mathrm{m}$ long. The mean mantle thickness corresponds in reality to a size distribution of thicknesses starting from zero. The packing factor of the particles is about 0.2 (80\% empty space) and leads to a mean mass density of $0.25 \mathrm{~g} \mathrm{~cm}^{-3}$ and an aggregate diameter of $5 \mathrm{\mu m}$. (Model constructed by Fred Robbers and photographed by Loek Zuijderduin).

EVOLUTION OF THE NUCLEUS IN THE OORT CLOUD AND LATER

Comets, once formed, become objects of the oort cloud circulating about the sun in near interstellar space. Insofar as further ultraviolet processing is concerned, even $4 / 2$ billion years can lead only to changes in the outer few microns (!) of the nucleus. Furthermore, the temperature of the nucleus, barring possible internal heating by radionuclides, is even lower than the 10-15 K of interstellar grains. However, cosmic ray protons are another matter and their effects have been considered by several authors $/ 32,33,34,35 /$. Their principal effect is to change the chemical composition of the material they penetrate in a way similar to the changes induced by ultraviolet processing of dust in interstellar space. The question is how significant is this additional processing?

It had already been shown by Donn and Moore that only the outer fraction of a meter of the nucleus could be chemically modified by the $\mathrm{MeV}$ cosmic ray protons so that the higher energy ones must first be slowed during penetration before they are effective. A recent calculation of the radiation dosimetry of a cometary nucleus by cosmic ray protons with energies ranging from $1 \mathrm{MeV}$ to $10^{10} \mathrm{GeV}$ which includes nuclear collision energy loss (not included in Ref. 35) as well as all secondary cascade effects $/ 36 /$ will be used as a comparison with the 
ultraviolet radiation dosimetry of interstellar grains. According to the new calculation the absorbed dose after $4 \frac{1}{2}$ billion years at about $1 \mathrm{~m}$ depth in a comet of density $1 \mathrm{~g} \mathrm{~cm}^{-3}$ is $10^{11} \mathrm{rad}$ which is quite small compared with the typlcal ultraviolet interstellar photoprocessing dose of $6 \times 10^{16} \mathrm{rad}$ on a core-mantle grain in its lifetime. Thus the ultraviolet dose on grains during only one of the several billion years they spend before aggregation is about $2.5 \times 10^{16}$ rad which is 100,000 times more than the cosmic ray dose at the surface after aggregation and even more in the interior. A range of comparisons is shown in Table 2. Since the outer few meters of a comet are lost already in its first apparition there is no reason to expect to find evidence of cosmic ray effects on a comet like Halley which has been around many times. However the production of a "crust" of some significance by cosmic ionizing particle effects does appear plausible. Its degree of permanence is not however established particularly in a comet which has been around as often as Halley. What crust we see now could perhaps have been produced in te solar system but not by comsic ray particles.

The next question is the modification of the nucleus by internal heating after entering the inner solar system. Heat conduction of porous structures is substantially lower than that of solid material. First of all we start with the fact that the grain ices are amorphous which means that their thermal conductivity is already a few orders of magnitude lower than crystalline ice $137 \%$. Next I have estimated the thermal conductivity of the structure shown in Fig. 2 by letting each grain have about $4-5$ grains touching it and by letting each contact surface be about $2 \times 10^{-15} \mathrm{~cm}^{2}$ (limited by local neat dissipation after collisions). Using a mean grain area of $2 \times 10^{-13} \mathrm{~cm}^{2}$ (fully "grown" pre-solar grain) means that only about 5 $x 10^{-2}$ of the surface of each grain can conduct heat so that the net heat conductivity of a comet is expected to be $\leq 5 \times 10^{-5}$ that of crystalline ice. This leads to a very high temperature gradient at the nucleus surface and at the same time, because of the very open structure, a very high evaporation rate which leads to efficient cooling. A detailed calculation incorporating both of these effects has not yet been done but it appears possible that while the surface of such a comet would be hotter than had been anticipated the amount of internal heating may still be low. The diffusion of vapor into the interior is limited by the very small pore size (recondensatiom occurs quickly) so that the morphological structure of the aggregated dust tends to be self preserving.

Table 2: The cosmic ray ionizing particle energy dose on a comet nucleus accumulated after

formation compared with the interstellar ultraviolet radiation dose before aggregation

into the nucleus.

\begin{tabular}{|c|c|c|c|c|c|c|}
\hline \multicolumn{2}{|c|}{ Depth (meters) } & \multirow{4}{*}{$\begin{array}{l}\text { C.R. dose } \\
4 y_{2} \times 10^{9} \mathrm{yr} . \\
\text { (Oort Cloud) } \\
\text { (Mrad) }\end{array}$} & \multicolumn{4}{|c|}{ (Oort cloud C.R. dose)/(Interstellar U.V. dose) } \\
\hline & & & $2 \frac{1}{2} \times 10^{9} \mathrm{yr}$ & $2 \frac{1}{2} \times 10^{9} \mathrm{yr}$ & $10^{8} \mathrm{yr}$ & $10^{8} \mathrm{yr}$ \\
\hline$\rho=1$ & $\rho=0.2$ & & (Diff. Cloud) & $(\mathrm{Mol} . \mathrm{Cloud})^{*}$ & (Diff. Cloud) & Mol. Cloud ${ }^{*}$ \\
\hline $8 \mathrm{~cm}^{-3}$ & $8 \mathrm{~cm}^{-3}$ & & & & & \\
\hline 0.1 & 0.5 & $3 \times 10^{5}$ & $(-6)$ & $(-4)$ & $1.25(-4)$ & $1.25(-2)$ \\
\hline 1 & 5 & $10^{5}$ & $1.7(-6)$ & $1.7(-4)$ & $(-5)$ & $(-3)$ \\
\hline 5 & 25 & $10^{4}$ & $1.7(-7)$ & $1.7(-5)$ & $(-6)$ & $(-4)$ \\
\hline 10 & 50 & $3 \times 10^{3}$ & $(-8)$ & $(-6)$ & $1.25(-7)$ & $1.25(-5)$ \\
\hline
\end{tabular}

* Mean reduction of ultraviolet radiation by $10^{-2}$ in overall molecular cloud phase. This corresponds to a 1 parsec thick cloud of density $\mathrm{n}_{\mathrm{H}}=5 \times 10^{3} \mathrm{~cm}^{-3}$. 


\section{a) Albedo and surface properties}

Both Vega and Giotto measures of the brightness of the surface of comet Halley outside the jets indicated very low albedos. According to Sagdeev et al./38/ the television system (TVS) aboard Vega 1 and $d 2$ gave $A-0.04+0.02$. The images obtained by the Halley multicolor camera aboard Giotto gave A $<0.04$. These values are consistent with the albedo derived for the open structure shown in Figure $2 / 30,39 /$. Because the individual grains have strongly forward throwing scattering phase functions and are somewhat absorbing, light penetrates the comet surface and is absorbed by multiple scattering within. A very good approximation to the surface albedo of a loosely agglomerated structure of particles with scattering asymmetry factor $\mathrm{g}(\mathrm{g}=$ mean value of the cosine of the scattering angle $=$ $\langle\cos \theta\rangle$ ), when $\mathrm{g} \rightarrow 1$, and individual particle albedo $\alpha(\alpha=$ scattering/absorption + scattering) is $A=\alpha(1-g) / 2$. For the particles shown in Fig. 2 one expects $g=0.8-0.9$ and $\alpha=0.5-0.6$ so that one gets $A=0.06-0.025$ which is about what is observed. It is of course possible and, in fact, likely that the actual surface micro structure of a comet is not simply as pictured in Fig. 2 but there seems no coupelling reason in view of the predicted albedo to expect it to be greatly modified except perhaps by the obvious effects of evaporation and of resetilng of previously ejected dust.

It has been suggested, alternatively, that the comet has a strongly absorbing crust which has been produced by cosmic ray bombardment in the oort cloud. Such a crust can not be as thick as that proposed by Gombosi and Houpis /40/ because, as noted by Sekanina /41/, the nucleus of comet Halley appears to be an extremely dynamic body, with the number of activity centers opening and closing on a time scale of one day or a fraction thereof. In view of the -52 hour rotation period confirmed by suizei $/ 42,43 /$ and the erratic behaviour of even the more enduring vents $/ 44 /$, a yielding surface is more likely than a rigid one. This is in line with the result of the calculation in which we have shown that whatever cosmic ray effects there may have been in the Oort cloud, they were limited to a layer which has long since been eroded away.

The evidence that Comet Halley activity is confined to the sunlit side /45/ suggests that the heat is limited to the surface and that little residual rise in temperature persists in the shadow region. A low internal heating also appears to be supported by the low nuclear spin temperature of $\mathrm{H}_{2} \mathrm{O}$ derived from the rate of the ortho and para transitions of neutral water near $2.65 \mathrm{\mu m} / 46 /$. The low thermal conductivity and low albedo are also supported by the high surface temperature of $300-400 \mathrm{~K} / 38,47 /$.

\section{b) Particle sizes}

The SP-2 experiment carried by the Vega spacecraft $/ 38,48 /$ as well as the dust impact detection system (DIDSY) on the Giotto spacecrafts gave evidence for large numbers of previously "unexpected" submicron sized particles down to the limit of detection at $10^{-17} \mathrm{~g}$ as well as particles in the $10^{-14} \mathrm{~g}$ range. Both of these sizes are expected and were indeed "predicted" from the size distribution of interstellar grains $/ 9,49 /$. As a result of heating, the ices evaporate and release the previously trapped hundredth micron sized particles some of which themselves may be heated and evaporated or eroded if they are of the carbonaceous type proposed here for the $220 \mathrm{~nm}$ hump of the interstellar extinction. A typical mean mass for the larger $(0.15 \mu \mathrm{m})$ individual interstellar grains (after ice mantle evaporation) is about $1.5 \times 10^{-14} \mathrm{~g}$ and for the $\mathrm{small}(\sim 0.01 \mu \mathrm{m})$ carbonaceous or silicate grains is about $10^{-17} \mathrm{~g}$.

The fact that there are hundredth micron sized particles which can be released from the icy grain mantles seems to preclude a strong chemical modification and polymerization produced by cosmic rays after grain aggregation. If such extra radiation were to occur, the carbonaceous and silicate hundredth micron particles, rather than simply being trapped in ices would, instead, be chemically bonded in a polymerized material and, having lost their individuality, would not reappear as observed in the size spectrum of Halley dust.

The spatial and temporal distribution of the masses and flux of dust particles measured by the dust counter and mass analyzer (DUCMA) on Vega $1 / 2$ showed, among other things, that 
the lowest masses were the first particles encountered at the fringes of the coma. One of the explanations for this phenomenon by simpson et al. $/ 44 /$ is that some of the dust particles are "comprised of much smaller particles" from which pieces are shed which appear at great distances as the material which binds them sublimes; i.e., the initial dust is clumpy.

The density of the dust particles deduced by Krasnopolsky et al $/ 50 /$ is about 0.35 $8 \mathrm{~cm}^{-3}$. Although this also supports the clumpy structure it is not quite as low as the density of the clumps shown in Fig. 3 which even with the volatiles included is $-0.25 \mathrm{~g} \mathrm{~cm}^{-3}$ but without the volatiles (a more likely case here) is only $0.12 \mathrm{~g} \mathrm{~cm}^{-3}$.

\section{c) Mass spectra of dust}

The dust impact mass analyzers on vega $1 / 2$ (PUMA) and on Giotto (PIA) showed a predominance of the light elements $\mathrm{H}, \mathrm{C}, \mathrm{O}, \mathrm{N}$ (organics) relative to the heavier elements $\mathrm{Si}, \mathrm{Mg}, \mathrm{Fe}$ (rockies) in the dust $123,24 \%$. There is considerable variety among the mass spectra although $80 \%$ of the particles were indeed dominated individually by the organics. Many of these are like those which would consist of the relative proportion of organics to silicate in the core-mantle interstellar grains. One should bear in mind that the organic refractory mantles have a range of possible thicknesses from zero to perhaps $0.2 \mu \mathrm{m}$ so that the light to heavy element ratio for individual $\left(10^{-14} \mathrm{~g}\right)$ grains ranges from -1 to significantly greater than $10: 1$. However clumps of dust $\left(210^{-13} \mathrm{~g}\right)$ which better represent an average composition would give a light to heavy ratio of about $10: 1$ in this model.

There are some spectra which could be characterized as pure $\mathrm{H}, \mathrm{C}, \mathrm{N}, \mathrm{O}$ and others as pure silicates ( $\mathrm{Si}, \mathrm{Mg}, \mathrm{Fe}, \mathrm{O}$ ). Until futher analysis of the data is forthooming it seems plausible to suggest that the pure $\mathrm{H}, \mathrm{C}, \mathrm{N}, \mathrm{O}$ grains correspond to the $220 \mathrm{~nm}$ hump particles and that the pure silicate particles correspond to the FUV interstellar particles.

An apparent anomaly exists when one compares the observed dust to gas production ratio of $Q_{\text {dust }} / Q_{\text {gas }}=0.25-0.1 / 38,48,51 /$ with that predicted $/ 26 /$ for dust which is predominantly organic. The latter gives a value of $Q_{\text {dust }} / Q_{\text {gas }}=0.65$ which is consistent with comets Arend Roland 1957III $(0.8 \pm 0.2)$ and with comet Bennet 1970II $(0.6 \pm 0.4)$ (Ref. 52). Even without an organic refractory component the predicted value is about 0.25 but then why is the organic component of dust predominant in the mass spectra? Perhaps closes to the comet surface the dust to gas ratio is higher (i.e. some of the organics are not so refractory and evaporate slowly) or perhaps there are more larger particles than have been included in the total dust mass calculation.

d) Dust contributions to the comet atmosphere.

Photographic observations of comet Halley's faint sunward spike have been analyzed by Sekanina $/ 41 /$ to show that it indicates the presence of extremely small (<< $0.1 \mu \mathrm{m}$ ) dielectric or nearly dielectric grains. These he associates with the prominent spectral signature of $\mathrm{CN}$ seen in the jet even before the scattered light by the spike was detected. Although the formation mechanism for the cyano radical is not certain it is virtually certain that the $\mathrm{CN}$ jets were formed in situ which suggests that they arise from molecules which are sublimating from the very small grains. The carbonaceous grains which we have proposed for the $220 \mathrm{~nm}$ hump have both the size and the chemical composition (cyanopolyenes, in part) to be the source of such molecules.

The ion mass spectrometer on board Giotto detected, among others, a surprisingly large abundance of the $\mathrm{C}^{+}$ion throughout the coma (Baisiger et al. /53/). The suggestion made by the author was that small carbonaceous particles heated in the coma could be relasing carbon atoms or highly unsaturated molecules providing a distributed source for photolysis and ionization leading to the $\mathrm{C}^{+}$. The source of the carbon molecules could be, in part, the hundredth micron $220 \mathrm{~nm}$ hump interstellar (carbonaceous) particles or the organic refractory mantle material onm the core-mantle grains. 
e) Infrared evidence for an organic refractory component of dust.

The infrared sounding of Comet Halley from Vega $1 / 47 /$ revealed spectral emission at $3.3 \mu \mathrm{m}$ and $7.5 \mu \mathrm{m}$ attributed to $\mathrm{C}-\mathrm{H}$ and $\mathrm{C}-\mathrm{C}$ bonds which could be related to the presence of carbonaceous material. Ground based infrared emission spectra of the $3.4 \mu \mathrm{m}$ band by Baas et al. /54/ and by Wickramasinghe and Allen /55/ have been interpreted as being caused by either very small solid organic refractory particles or by solid particles plus large molecules. The detailed structures shown in ref. 54 probably provide the best available means of identifying the organics. It is important to note the need for the sources of emission "structures" to be very small in order to make the $3.4 \mu \mathrm{m}$ emission possible.

\section{f) Molecule and ion components in the gaseous coma.}

Of course, $\mathrm{H}_{2} \mathrm{O}$, was the most abundant molecule deduced in the coma of comet Halley. The next most abundant species are probably $\mathrm{CO}_{2}$ and $\mathrm{CO}$. For example Krankowsky et al. /56/ found a ratio $\mathrm{Q}_{\mathrm{CO}} / \mathrm{Q}_{\mathrm{H}_{2} \mathrm{O}}=0.03$ and infrared data $/ 47 /$ gave $\mathrm{Q}_{\mathrm{CO}_{2}} / \mathrm{Q}_{\mathrm{H}_{2} \mathrm{O}}=10^{-2}$ while IUE observations $157 /$ gave $Q / Q_{H}=0.1-0.2$. These values are more or less within the ranges suggested by the $\mathrm{COlat}$ fle composition of interstellar dust $/ 58,59 /$ while, at the same time, there is no definite evidence for the presence of $\mathrm{NH}_{3}$ in the ion mass spectra 153\%. Although $\mathrm{NH}_{3}$ has been suggested to be a substantial component of interstellar dust the observational evidence has become less compelling, while theoretical arguments lead to generally rather smaller amounts of $\mathrm{NH}_{3}$ in grain mantles than $10 \% / 2 \%$

It was noted by Balsiger et al. $/ 53 /$ that the $\mathrm{C} / 0$ ratio is about half of the cosmic abundance ratio. This has earlier been called the missing carbon mystery by Delsemme /52/ and has been attributed to the "hiding" of a large fraction of the carbon in the organic refractory component $/ 26 /$.

g) Density of comet Halley.

Now that the nucleus of comet Halley has finally been directly seen and its volume measured it has become possible to make proper estimates of its mass density. An earlier estimate of the size of comet Halley was as a sphere of radius $=2.4 \mathrm{~km}$ and a density

$\geq 1 \mathrm{~g} \mathrm{~cm}^{-3} / 60 \%$. Using the current size estimate as that of a prolate spheroid of diameter $\overline{7} \mathrm{~km}$ and length $15 \mathrm{~km}$ the volume is about 6 times larger leading to a mass density $\rho>0.17 \mathrm{~g} \mathrm{~cm}^{-3}$ which is in the range of $\rho=0.25 \mathrm{~g} \mathrm{~cm}^{-2}$ derived from the interstellar dust configuration in Fig. 2.

\section{CONCLUSIONS}

The key questions raised and discussed have been: are comets born as aggregated interstellar dust and what evidence do we have that comet nuclei maintain or do not maintain their initial composition and structure?

The cold formation of comets out of interstellar dust has been reinforced by the demonstration that not only is pre-aggregation ultraviolet radiation of sulfur-containing interstellar grain mantles adequate to produce the $S_{2}$ as a parent molecule but that calculated values of cosmic ray ionizing particle penetration of the nucleus in the oort cloud phase are not sufficient to do so except in the outer meter or so. The presence of abundant very small $\left(\leq 10^{-17} \mathrm{~g}\right.$ ) particles in the dust size spectrum of comet Halley is a confirmation that the penetration of cosmic ray protons in the nucleus has not substantially modified or polymerized the comet ices to the depths observed and that if any such modification had existed it has long since been eroded away.

The low density and morphological structure, on a microscale, predicted by the aggregated dust model are consistent with the low albedo and low thermal conductivity of the comet Halley surface. The high surface temperature, the preponderance of dust/gas jets on the sunward side, and the low nuclear spin temperature states of the $\mathrm{H}_{2} \mathrm{O}$ provide evidence for a high surface temperature gradient and a low heat penetration. This indicates that the morphological structure of aggregated dust may be self preserving even in the apparition phase. 
The mass spectra and infrared emission spectra of the dust show that the major component of the solids is an organic refractory material similar to that observed in interstellar dust. For the reasons stated above this can not have been produced in the nucleus during the oort cloud phase.

The evidence provided by comet Halley results is strongly in favor of the serious consideration of comets being very much like original aggregates of interstellar dust except possibly the outermost surface layers. The fluffiness of the comet gives rise to the possibility that a large fraction of its submicron components of refractory organic material could be ablated during passage of the comet through the earth's atmosphere and float down to the surface preserving the organic component in its original form. This implies the possibility that early comet impacts on the earth could have supplied the interstellar organics as the first abundant building blocks for the origin of life.

\section{REFERENCES}

/1/ J.M. Greenberg, 1985 in "Birth and Infancy of Stars, eds. R. Lucas, A. Omont and R. Stora (Elsevier), 139-203.

/2/ L.B. d'Hendecourt, L.J. Allamandola, J.M. Greenberg, 1985, Astron. Astrophys., 152, 130-150.

13/ L.B. d'Hendecourt, L.J. Allamandola, R.J.A. Grim, J.M. Greenberg, 1986, Astron. Astrophys., 158, 119.

/4/ J.M. Greenberg, G.C. Chlewicki, 1983, Astrophys. J., 272, 563.

/5/ G.C. Chlewicki, 1985, Ph. D. Thesis U. of Leiden.

16/ J.M. Greenberg, 1968, in "Stars and Stellar Systems" vol. VII (eds. B.M. Middlehurst, and L.H. Aller) (Univ. of Chicago Press) 221-364.

/7! T.P. Stecher, 1965, Astrophys J., 142, 1683.

/8/ J.M. Greenberg, 1982, in "Submillimetre Wave Astronomy" eds. D. Phillips and J.E. Beckman (Cambridge U. Press) 161.

19/ J.M. Greenberg, 1982, in "Comets" ed. L.L. Wilkening (U. of Arizona Press), 131-163.

/10/ V.K. Agarwal, W. Schutte, J.M. Greenberg, J.P. Ferris, R. Briggs, S. Connor, C.P.E.M. Van de Bult and F. Baas, 1985, Origins of Life, 16, 21-40.

/11/ S.P. Willner, R.W. Russell, R.C. Puetter, B.T. Solfer and P.M. Harvey, 1979, Astrophys. J., 229, L65-L68.

/12/ I. Butchart, A.D. MoFadzean, D.C.B. Whittet, T.R. Geballe, J.M. Greenberg, 1986, Astron. Astrophys. 154, 15.

/13/ J.M. Greenberg, in "Laboratory and Observational Infrared Spectra of Interstellar Dust", 1984, eds. R.D. Wolstencroft and J.M. Greenberg, Occasional Reports of the Royal Obs. Edinburgh ISSN0309-049X, 82-92.

/14/ W. Schutte and J.M. Greenberg, 1986, in "Light on Dark Matter", ed. F.F. Israel (Reidel), 229-232.

115/ J.A. Nuth, 1985, Nature, 318, 116.

116/ R.D. Savage, J.S. Mathis, 1979, Ann. Rev. Astron. Astrophys. 17, 73.

/17/ S.S. Hong and J.M. Greenberg, 1980, Astron. Astrophys., 189. J.S. Mathis, W. Rumpl, and K.H. Nordsleck, 1977, Astrophys. J., 217, 425. 
118/ G.P. Van der Zwet, M.S. de Groot, F. Baas, and J.M. Greenberg, 1986, in "Polycycllc Aromatic Hydrocarbons and Astrophysics", ed. A. Leger and L.B. d'Hendecourt, in press.

/19/ J.M. Greenberg, M.S. de Groot and G.P. van der Zwet, 1986, in "Polycyclic Aromatic Hydrocarbons and Astrophysics", ed. A. Leger and L.B. d'Hendecourt, in press.

/20/ B.D. Savage, 1975, Astrophys. J., 188, 92.

/21/ T.P. Snow and C.G. Seab, 1980, Astrophys. J. 242, L83.

122/ J.M. Greenberg, 1978, in "Cosmic Dust" ed. J.A.M. MCDonnell (W1ley, N.Y.), 187-294.

/23/ J. Kissel et al. Nature, 321, 280.

/24/ J. Kissel et al. Nature, 321, 336.

/25/ J.M. Greenberg, R. Grim and L. van IJzendoorn, 1986, in Asteroids, Conets, Metors, eds. C.I. Lagerkvist, B. Lindblad, H. Lundstedt and H. Rickman, Reprocentrum HSC, Uppsala Press (1986) 218-220.

/26/ J.M. Greenberg, 1983, in "Cometary Exploration" ed. T.I.Gombosi (Hungarian Academy of Sciences) 23.

/27/ F. Verniani, 1969, Space Science Reviews 10, 230-261.

128/ F. Verniani, 1973, J. Geophys. Res. 78, 8429-8462.

/29/ P.M. Millman, 1976, in "Interplanetary Dust and Zodiacal Light" eds. H. Elstisser and H. Fechtig (Springer-Verlag) 359-372.

/30/ J.M. Greenberg, 1986, in "Asteroids, Comets and Meteors II, eds. C.-I. Lagerkvist, B.A. Lindblad, H. Lundstedt and H. Rickman, (Uppsala University Press) 221-223.

/31/ Z. Sekanina and D.K. Yeomans, 1985, Astron. J. 2335.

132/ R.E. Johnson, L.J. Lanzerotti, W.L. Brown, W.M. Augustiniak, and C. Mussil, 1983, Astron. Astrophys. 123, 343.

/33/ F. Whipple, 1977, in "Comets, Asteroids, Meteorites", ed. A. Delseme (U. of Toledo), 25 .

134/ M.H. Moore and B. Donn, 1982, Astrophys. J. 257, L47.

135/ G. Strazzulla, 1986, I carus, 67, 63-70.

/36/ M.P. Ryan Jr. and I.G. Draganic, 1986, Astrophys. Sp. Sc1. In Press.

/37/ J. Klinger, 1980, Science 209, 271-272.

/38/ Sagdeev et al. 1986, Nature, 321, 259.

/39/ Greenberg, J.M. 1986, Nature, 321, 385.

140/ T.I. Gombosi and H.L.E. Houpis, 1986, Nature, submitted.

/41/ z. Sekanina, 1986, Adv. in Space Res., to be published.

/42/ E. Kaneda et al. 1986, Nature, 320, 140.

/43/ Z. Sekanina and S.M. Larson, 1986, Astron. J., in press. 
/44/ J.A. Simpson et al. 1986, Nature, 321, 278.

145/ Z. Sekanina and S.M. Larson, 1986, Nature, 321, 357.

/46/ M.J. Mumma, H.A. Weaver, H.P. Larson, D.S. Davis, M. Williams, 1986, Science, 232, 1523.

/47/ M. Combes et al. 1986, Nature, 321, 266.

/48/ E.P. Mazets et al. 1986, Nature, 321, 276.

/49/ J.M. Greenberg, 1984, Adv. Space Res., 4, 211.

/50/ V.A. Krasnopolsky et al. 1986, Nature, 321, 269.

151/ J.A.M. McDonnell et al. 1986, Nature, 321, 338.

/52/ A.H. Delsemme, 1982, in "Comets", ed. L.L. Wilkening (U. of Arizona Press) 85.

/53/ H. Balsiger et al. 1986, Nature, 321, 330 .

/54/ F. Baas, T.R. Geballe and D.M. Walther, 1986, Astrophys. J. Letters, in press.

155/ D.T. Wickramsinghe and D.A. Allen, 1986, Nature, 323, 44.

156/ D. Krankowsky et al. 1986, Nature, 321, 326.

157/ M.C. Festou et al. 1986, Nature, 321, 361.

/58/ D.C.B. Whittet, A.J. Longmore, A.D. McFadzean, 1985, MNRAS, 216, 45p.

/59/ J.M. Greenberg, 1983 in "Asteroids, Comets, Meteors, eds. C.I. Lagerkvist and H. Rickman, (Uppsala University Press) 259.

/60/ Belton, M.J.S. 1985, Science, 230, 1229-1236. 